\title{
Damping Characteristics of Carbon Nanotube-Epoxy Composites via Multiscale Analysis
}

\author{
Ailin $\mathrm{Liu}^{1}$ and K.W. Wang ${ }^{2}$ \\ University of Michigan, Ann Arbor, MI, 48109 \\ Charles E. Bakis ${ }^{3}$ \\ The Pennsylvania State University, University Park, 16802
}

In this paper, the damping characteristics of epoxy resin containing aligned or randomly oriented carbon nanotube (CNT) ropes are investigated via a multiscale analysis approach. The shear strengths at the inter-tube and tube-resin interfaces are calculated using molecular dynamics simulations of nanotube pullouts before being applied to a micromechanical damping model. In the micromechanical model, the composite is described as a three-phase system composed of a resin, a resin sheath acting as a shear transfer zone, and a carbon nanotube rope. The concept of stick-slip motion is used to describe the load transfer behavior between carbon nanotubes in a rope as well as between nanotubes and the surrounding sheath. Both the energy dissipations from the viscoelastic polymer matrix and from the stick-slip motion are included in the overall structural damping characteristics. The effect of nanorope alignment on damping characteristics is also presented.

\section{Nomenclature}

$E_{1} \quad=$ Young's modulus in three-element standard solid model for viscoelastic resin

$E_{2} \quad=$ Young's modulus in three-element standard solid model for viscoelastic resin

$E_{e q} \quad=$ equivalent Yong's modulus for carbon nanotube as a solid cylinder

$f_{r p} \quad=$ volume fraction of carbon nanotube rope

$\bar{G} \quad=$ complex shear modulus of viscoelastic resin

$G_{e q} \quad=$ equivalent shear modulus for carbon nanotube as a solid cylinder

$K=$ bulk modulus of viscoelastic resin

$L_{a} \quad=$ length of composite unit cell

$L_{n t} \quad=$ length of carbon nanotube

$l_{t-s}^{e f f} \quad=$ effective length of SWNT/sheath debonding

$R_{n t} \quad=$ radius of carbon nanotube

$R_{r p} \quad=$ radius of carbon nanotube rope

$R_{s h} \quad=$ outer radius of the sheath

$t_{a} \quad=$ thickness of composite unit cell

$\tan \delta \quad=$ total effective loss factor

$\tan \delta_{r s}=$ loss factor contribution from viscoelastic resin

$\tan \delta_{s h}=$ loss factor contribution from viscoelastic sheath

$\tan \delta_{\text {slip }}=$ loss factor contribution from stick-slip movement

$u_{r s} \quad=$ displacement of resin along tube direction

\footnotetext{
${ }^{1}$ Postdoctoral Fellow, Mechanical Engineering, 2271 G G Brown Laboratory

${ }^{2}$ Stephen P. Timoshenko Collegiate Professor of Mechanical Engineering, 2236 G G Brown Laboratory

${ }^{3}$ Distinguished Professor of Engineering Science and Mechanics, 212 Earth-Engineering Science Building 


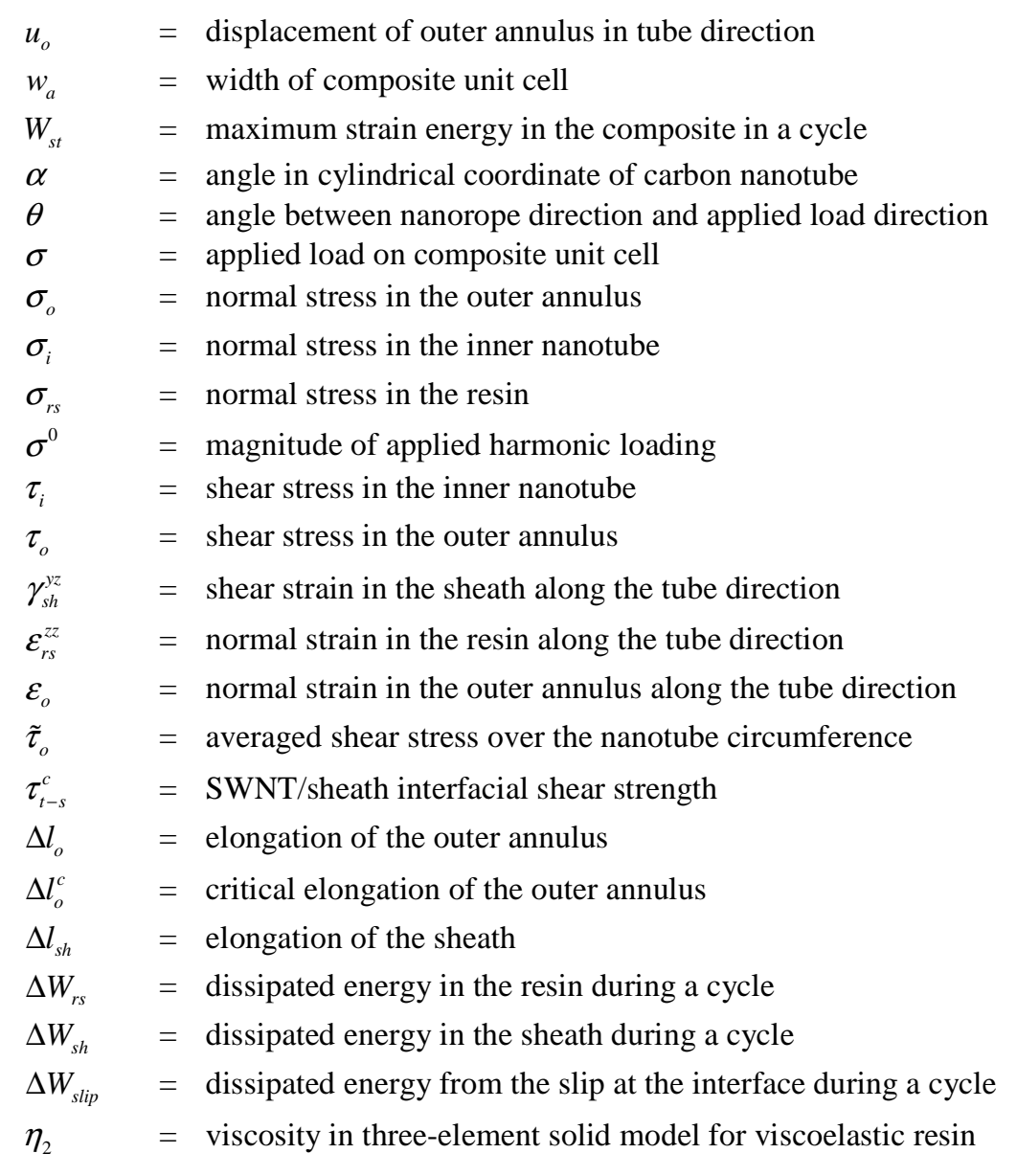

\section{Introduction}

NT-based composites have attracted increasing interest regarding their damping characteristics due to the exceptional features of carbon nanotubes. It was reported from experimental studies of composites with dispersed CNT fillers ${ }^{1,2}$ that the damping properties of polymeric materials increased by adding a small amount of carbon nanotubes. The loss factor of such composites was also found to be sensitive to the strain (stress) level. High damping can be achieved by taking advantage of the CNT-resin interfacial friction with the ultra large interfacial surface area between the nanotube and the resin. Applying the stick-slip phenomenon observed at the atomic scale on graphite surface ${ }^{3,4}$ to the nanotube/resin interface, Zhou et al. ${ }^{1}$ first developed a stick-slip damping model for composites containing aligned, well-dispersed individual single-wall nanotubes (SWNTs) under quasi-static monotonically increasing normal stress. The model was later expanded to composites with aligned nanoropes ${ }^{5}$ and randomly oriented nanoropes ${ }^{6}$. Since polymeric matrix materials possess viscoelastic properties that will be influenced by the nanotubes fillers and at the same time affect the overall damping, their characteristics have also been included in recent investigations ${ }^{7}$.

While the current damping models of CNT-based composites show great potential, it is recognized that more research efforts are needed to further advance the state of the art. For instance, the analyses in previous studies only focused on composites under quasi-static, monotonically increasing load. In actual applications, the damping materials are normally characterized, evaluated, and used under cyclic loading. With frictional slip at the interfaces, the unloading process in a cycle is complicated and results cannot be easily obtained by simply extending from the previous models derived for monotonically quasi-static loading conditions. Moreover, the studies discussed above $^{1,5-7}$ indicated that the interfacial CNT-CNT and CNT-resin shear strengths significantly affect the damping properties of CNT-based composites. However, in these previous investigations, the values of the interfacial shear strength were chosen arbitrarily based on results reported in other publications. In order to develop a comprehensive 
model to explore or design various different material systems, a multiscale approach that can characterize the CNT interfacial strength at the molecular/atomic level, the stick-slip phenomena at the microscopic level and the material damping feature for macroscopic applications is needed.

To advance the state of the art and eventually derive an effective analysis tool that can be used to synthesize CNT-based damping composites, the research presented in this paper addresses the aforementioned technical issues and develops a multiscale damping model for CNT rope-filled composites under cyclic loading.

\section{Modeling Approach}

In this study, a sequential two-part multiscale damping model is proposed. First, the interfacial shear strength is calculated by simulating a CNT pull-out test using the molecular dynamics method. Interfacial shear strengths between nanotube and polymer resin as well as between adjacent nanotubes in a rope are investigated. Strength values obtained from atomic simulations are then applied to a micromechanical damping model of a representative unit cell of a CNT/polymer composite. The micromechanical unit cell is modeled as a three-phase system composed of a resin, a resin sheath transferring load between the CNT and resin, and CNTs in the form of SWNT ropes. The sheath is a thin layer of the resin around the nanotubes which transfers only shear stress between the CNTs and the resin. For simulation convenience, the sheath is assumed to share the same material properties as the resin. The concept of stick-slip motion caused by interfacial friction is applied to characterize the interface between the nanoropes and resin as well as between individual nanotubes. Details of the multiscale damping model are presented in the following sections.

\section{A. Molecular dynamics simulations}

In the current investigation with MD simulation, the interfacial shear strength is calculated using the method derived from balance of momentum ${ }^{8}$. However, instead of the pull-through test discussed in Frankland's study, we modified the approach to emulate a pull-out test by eliminating the periodic boundary condition assumption used by Frankland. The Tersoff potential ${ }^{9}$ is used to describe the carbon nanotubes while the OPLS-UA potential ${ }^{10}$ is applied to the epoxy resin. All the molecular dynamics simulations are performed with the DL_POLY software package ${ }^{11}$.

Two types of pull-out tests are simulated: one pulls out one SWNT from a rope containing seven hexagonal-packed SWNTs; the other pulls out one SWNT embedded in epoxy resin. Fig. 1 shows the equilibrated systems for CNT-CNT pullout test and CNT-resin pullout test. The length of the SWNTs in a rope is $36 \AA$ including caps at both ends. The epoxy resin used in this study is EPON 862 and EPI-CURE W curing agent

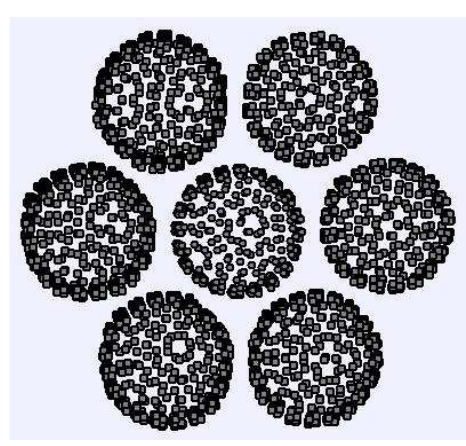

(a)

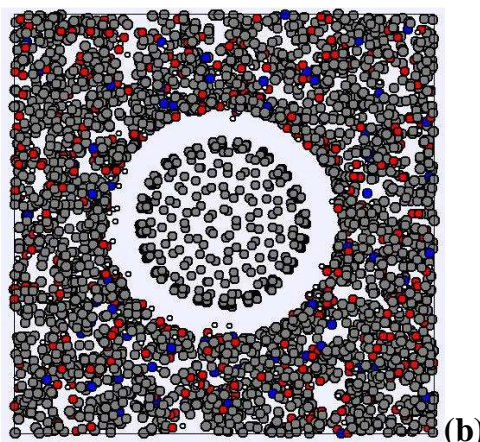

Figure 1. Top View of equilibrated system of (a) seven hexagonalpacked SWNTs (b) epoxy resin with one embedded SWNT. Grey dots represent carbon, red is oxygen, blue is nitrogen, small circle without filling is hydrogen (hydrogens on carbon are implicit). with weight ratio of 100:26.4. The

SWNT embedded in the epoxy resin is $53 \AA$ including caps at both ends.

In order to pull out the inner SWNT from a rope, after equilibration at $300 \mathrm{~K}$, all the atoms in the outer six CNTs are fixed while the external force is applied uniformly to each atom of the inner CNT. Figure 2 shows the externally applied force, average velocity, and average displacement of the inner CNT in the tube length direction as a function of simulation time during the tube-tube pullout scenario. When the external force is small, the inner CNT displays thermal vibration but no net motion. This phenomenon continues until the applied force increases to $0.54 \mathrm{nN}$, after which the inner tube starts to oscillate around a slightly displaced position. When the applied force is about $1.3 \mathrm{nN}$, the average velocity and displacement of the inner CNT start to increase dramatically, indicating complete pull-out of the inner tube. The interfacial shear strength is the ratio of the threshold force to the total interfacial surface area. For the case studied here, the interfacial shear strength between nanotubes in a rope is $27.7 \mathrm{MPa}$. 


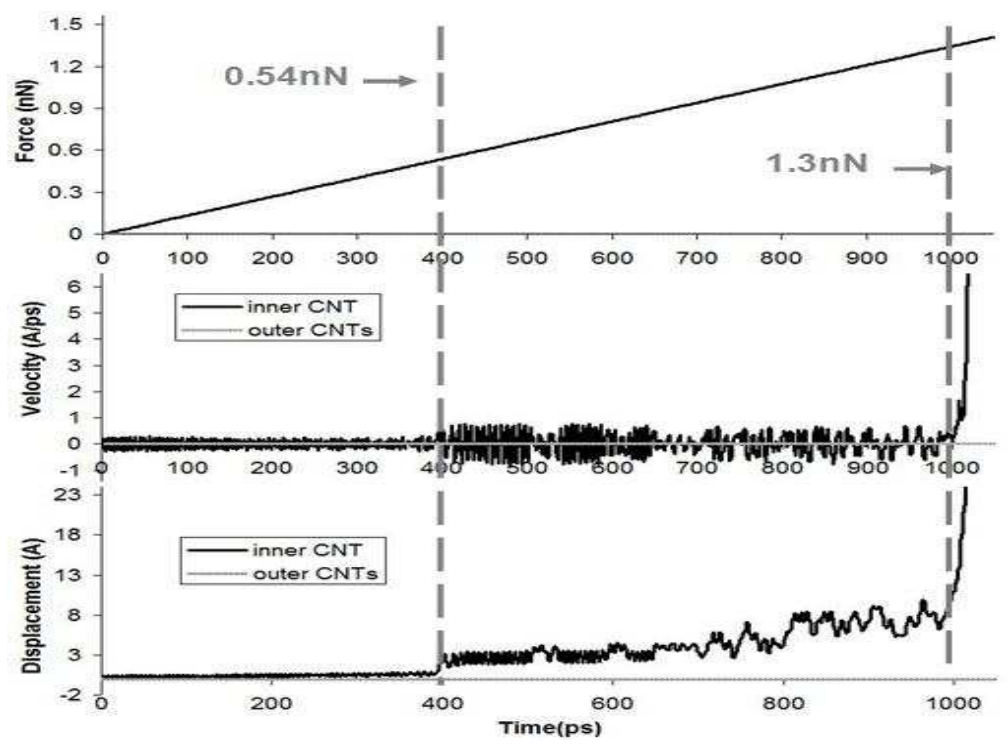

Figure 2. Simulated pullout of the inner CNT from a rope: (a) external force (b) average velocities and (c) average displacements of the inner and outer nanotubes.

included in the model due to the large aspect ratio of the nanotube.

In this study, seven identical SWNTs in a hexagonal arrangement are used to illustrate the nanotube rope. It is assumed that the six outer SWNTs comprise a monolithic annulus, as shown in Fig. 3 with dashed lines, implying that the normal and shear stresses in the six outer tubes are continuous. Therefore, only two types of load transfer are considered in such a SWNT lattice: one representing interactions at the interfaces between the outer annulus and the surrounding material, and the other representing interactions at the interfaces between the inner SWNT and outer annulus, as schematically illustrated in Fig. 3.

Similar to the three-phase model for polymeric composites with discontinuous fiber reinforcement ${ }^{13}$, the nanorope is assumed to be surrounded by a sheath of resin in a unit cell of the composite (Fig. 4).

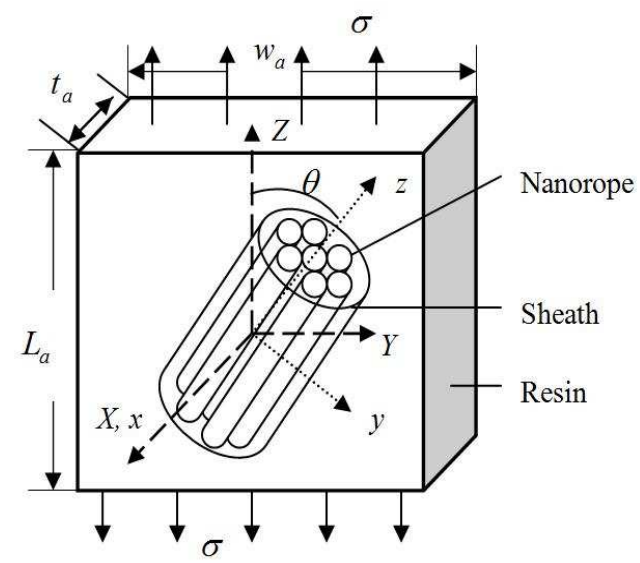

(a)

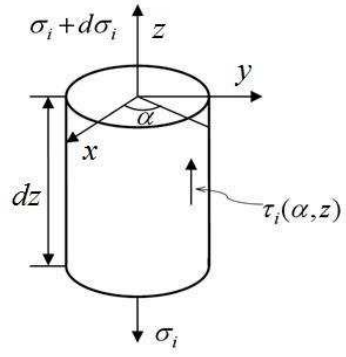

Figure 4. Representative volume element: (a) unit cell consisting of a bundle of seven SWNTs surrounded by a sheath and remote resin; (b) stresses on an element of length $d z$ in the inner CNT.
Similarly, the shear strength between the SWNT and the resin is $18.8 \mathrm{MPa}$ from pullout test. This result shows a fair match with the interfacial shear strength obtained from pulling MWNTs from epoxy polymer with an AFM cantilever, $30 \pm 7 \mathrm{MPa}^{12}$. For this case study, the calculated interfacial tube-resin shear strength is smaller than the inter-tube shear strength.

\section{B. Micromechanical damping model}

In the micromechanical damping model, the following basic assumptions are applied: (a) the carbon nanotube rope is straight and does not buckle under compression; (b) the carbon nanotube ropes are dilute in the resin; (c) only the normal and shear stresses oriented along the tube-length direction are

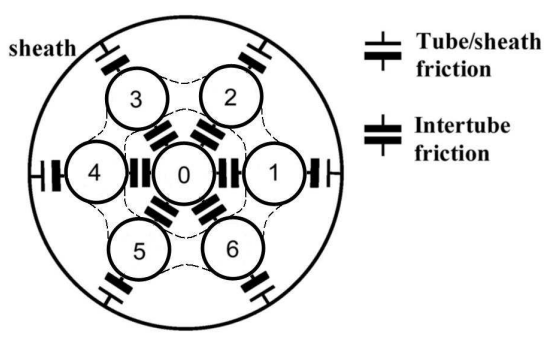

Figure 3. A schematic of load transfer in the tube lattice model

The nanotubes are assumed to have rotation angles only in the $Y-Z$ plane. The sheath carries only longitudinal shear stress between the nanotubes and the remote resin material; normal stress in the sheath is negligible. We also assume that the sheath shares the same material properties as the resin. The CNT-based composite is, thus, modeled as a three-phase composite: resin, sheath, and nanorope. The length, width and thickness of the unit cell are $L_{a}, w_{a}$ and $t_{a}$, respectively. These geometric parameters 
are related to those of nanotubes by the volume fraction of the nanorope in the composite, $f_{r p}$, according to the relationship

$$
\pi R_{r p}^{2} L_{n t}=f_{r p} L_{a} w_{a} t_{a}
$$

where $R_{r p}$ and $L_{n t}$ are the radius and length of the carbon nanotube rope, respectively.

Based on viscoelastic material theory ${ }^{14}$, a three-element standard solid model is adopted for the epoxy resin. Since the aspect ratio of the nanotube is large and the stick-slip motion is only considered in the longitudinal direction of the nanotubes, the response of the nanorope to applied longitudinal normal and shear stresses along the $\mathrm{z}$-axis is modeled using a viscoelastic element in parallel with a Coulomb friction.

In this model, a shear lag analysis is used to characterize load transfer in and around a nanorope lattice in the unit cell. The normal stresses on the inner SWNT and the outer annulus of SWNTs in the tube axial direction are represented by $\sigma_{i}$ and $\sigma_{o}$, respectively. The shear stresses acting on the inter-tube and SWNT/sheath surface are represented as $\tau_{i}$ and $\tau_{o}$, respectively, where $\alpha$ is the polar angle measured in $x-y$ plane of the nanorope as shown in Fig. 4(b). With the assumptions that the uniaxial normal stress in the resin is spatially uniform and that the normal stress in the sheath is negligible, the overall equilibrium along the $z$-direction of the unit cell as shown in Fig. 4(a) gives

$$
\pi R_{n t}^{2} \sigma_{i}+6 \pi R_{n t}^{2} \sigma_{o}+\left(\frac{w_{a} t_{a}}{\cos \theta}-\pi R_{s h}^{2}\right) \sigma_{r s}^{z z}=\sigma w_{a} t_{a} \cos \theta
$$

where $R_{n t}$ and $R_{s h}$ are the radius of the nanotube and the sheath; $\sigma$ is the applied stress on the composite in the $Z$ direction; and $\sigma_{r s}^{z z}$ is the normal stress in the resin along the $z$-direction.

The shear strain in the sheath, $\gamma_{s h}^{y z}$, is determined from the displacement of the outer annulus, $u_{o}$, and the resin, $u_{r s}$, along the $z$-direction:

$$
u_{r s}-u_{o}=\left(R_{s h}-R_{r p}\right) \gamma_{s h}^{y z}
$$

Taking the first derivative of Eq. (3) with respect to $z$ and using the strain-displacement relations and the stressstrain relations for the sheath, we obtain

$$
\varepsilon_{r s}^{z z}-\varepsilon_{o}=\frac{R_{s h}-R_{r p}}{\bar{G}} \frac{d \tilde{\tau}_{o}}{d z}
$$

where $\bar{G}$ is the complex shear modulus of the viscoelastic resin which can be obtained from three-element standard

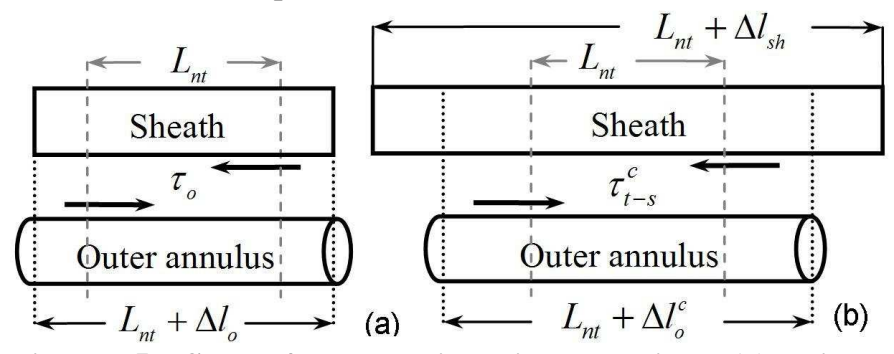

Figure 5. SWNT/sheath stick-slip behavior. (a) stick, $\tau_{o}<\tau_{t-s}^{c}, \Delta l_{s h}=\Delta l_{o}$ and (b) slip, $\tau_{o}=\tau_{t-s}^{c}, \Delta l_{s h}>\Delta l_{o}=\Delta l_{o}^{c}$. solid $\operatorname{model}^{12}, \varepsilon_{r s}^{z z}$ and $\varepsilon_{o}$ are the normal strains of the resin and the outer annulus in the $z$-direction, respectively.

The stick-slip motion is applied to the interface between the inner CNT and the outer annulus as well as between the outer annulus and the surrounding sheath. For instance, the outer annulus and the sheath are perfectly bonded if the interfacial shear stress $\tau_{o}$ is less than the SWNT/sheath shear strength $\tau_{t-s}^{c}$, as shown in Fig. 5(a). The

SWNT/sheath slippage occurs when the interfacial shear stress reaches the critical value. Once the debonding starts, the outer annulus and the sheath have different elongations $\Delta l_{s h}>\Delta l_{o}^{c}$ (Fig. 5(b)).

From shear lag analysis, the equilibrium of the stresses on the inner SWNT can be expressed as follows:

$$
\pi R_{n t} \frac{d \sigma_{i}}{d z}+\int_{0}^{2 \pi} \tau_{i} d \alpha=0
$$

For the outer annulus, the shear stresses include those from the inner SWNT $\tau_{i}$ and those from the sheath $\tau_{o}$ :

$$
\pi R_{n t} \frac{d \sigma_{o}}{d z}+4 \int_{0}^{2 \pi} \tau_{o} d \alpha-\int_{0}^{2 \pi} \tau_{i} d \alpha=0
$$


The normal stresses at the ends of SWNT are zero, and the average shear stresses over the nanotube circumference at the middle length of the nanorope $(z=0)$ are also zero, that is, $\int_{0}^{2 \pi} \tau_{i} d \alpha=\int_{0}^{2 \pi} \tau_{o} d \alpha=0$. In general, the boundary conditions for the inner SWNT and the outer annulus are

$$
\begin{gathered}
\left.\sigma_{i}\right|_{z= \pm L_{n t} / 2}=\left.\sigma_{o}\right|_{z= \pm L_{n t} / 2}=0 \\
\left.\tau_{i}\right|_{z=0}=\left.\tau_{o}\right|_{z=0}=-\sigma \sin \theta \cos \theta \sin \alpha
\end{gathered}
$$

By solving the above governing equations with numerical iterations, the strain distribution in each constituent of the composite unit cell can be obtained. The solution of the governing equations indicates that the inter-tube shear stress is smaller than the SWNT/sheath shear stress in a perfectly bonded system. Recall that the inter-tube shear strength is larger than the tube-resin shear strength from molecular dynamics simulation. Therefore, SWNT/sheath slip starts before inter-tube slip is initiated. Furthermore, for the case studied in this paper, energy is dissipated due to interfacial sliding only at the SWNT/sheath interface.

In cyclic loading situations, consider a fully-reversed (tension-compression) harmonic loading cycle with period of $T, \sigma=\sigma^{0} \sin (2 \pi t / T)$. Stresses and strains in all constituents are assumed to be zero at the start of the first cycle at zero applied stress. As loading begins, the composite undergoes a tensile stress which we assume is sufficient to cause debonding. When unloading begins, the nanorope is assumed to immediately stick to the resin as the strain of the resin decreases ${ }^{15}$. Thus, the stresses in the constituents and at the interfaces during the unloading process can be obtained by superposing the stresses existing at the previous peak strain and, at first, the compressive elastic stresses in relation to the previous peak strain. If the applied stress magnitude is sufficiently large, slipping can occur on the compression side of the loading cycle.

Using the calculated stress and strain distribution obtained under cyclic loading, the damping characteristics of the CNT-based composite unit cell can be obtained via the loss factor. The effective loss factor is defined as the ratio of the dissipated energy per unit volume to the maximum stored energy per unit volume ${ }^{16}$. There are three mechanisms of energy dissipation in the composite containing dilute nanoropes: one is the energy loss due to the stick-slip motion; the other two are the energy dissipations in the viscoelastic resin and sheath, respectively. The effective loss factor of the composite containing nanoropes is:

$$
\tan \delta=\left(\Delta W_{r s}+\Delta W_{s h}+\Delta W_{\text {slip }}\right) / W_{s t}=\tan \delta_{r s}+\tan \delta_{s h}+\tan \delta_{\text {slip }},
$$

where $W_{s t}$ is the maximum strain energy in the entire composite in a period. To determine energy loss due to the stick-slip motion, Kelly ${ }^{17}$ proposed that the dissipated energy due to friction is equivalent to the frictional shear force times the differential displacement at the interface. In the present model, the energy dissipation associated with stick-slip motion between the outer annulus and the resin is:

$$
\Delta W_{\text {slip }}=\int_{\text {cycle }} \tau_{t-s}^{c} \cdot 8 \pi R_{n t}\left(l_{t-s}^{e f f}\right)^{2}\left(d \varepsilon_{r s}-d \varepsilon_{o}\right),
$$

\begin{tabular}{|c|c|c|}
\hline Unit cell & Length, $L_{a}$ & $1.5 \mu \mathrm{m}$ \\
\hline \multirow{4}{*}{$\begin{array}{l}\text { Resin } \\
\text { (Three-element } \\
\text { standard solid } \\
\text { model) }\end{array}$} & Young's modulus, $E_{1}$ & $3.3 \mathrm{GPa}^{*}$ \\
\hline & Young's modulus, $E_{2}$ & $3.3 \mathrm{GPa}^{*}$ \\
\hline & Viscosity, $\eta_{2}$ & $50 \mathrm{GPa} \cdot \mathrm{s}$ \\
\hline & Bulk modulus, $K$ & $3.67 \mathrm{GPa}$ \\
\hline \multirow[t]{2}{*}{ Sheath } & Inner radius, $R_{r p}$ & $2.544 \mathrm{~nm}$ \\
\hline & Outer radius, $R_{s h}$ & $10.2 \mathrm{~nm}$ \\
\hline \multirow{3}{*}{$\begin{array}{l}(10,10) \\
\text { SWNT }\end{array}$} & Equivalent Young's modulus, $E_{e q}$ & $641.1 \mathrm{GPa}^{\dagger}$ \\
\hline & Equivalent shear modulus, $G_{e q}$ & $38.6 \mathrm{GPa}^{\dagger}$ \\
\hline & Radius, $R_{n t}$ & $0.848 \mathrm{~nm}$ \\
\hline \multicolumn{3}{|c|}{$\begin{array}{l}\text { The definition of the three parameters is given in Ref. } 12 \\
\text { The equivalent Young's and shear moduli of SWNT as a solid } \\
\text { cylinder are obtained following the effective fiber procedure in } \\
\text { Ref. } 18 \text {. }\end{array}$} \\
\hline
\end{tabular}

where $l_{t-s}^{e f f}$ is the effective length of the SWNT/sheath slippage.

\section{Results and Discussion}

Table 1. Material properties used in the model

6
The derived multiscale damping model can be used to analyze the loss factor for composites containing aligned or randomly oriented dilute MWNTs or SWNT ropes. All the material properties used in this simulation are listed in Table 1. Recall that inter-tube sliding will not occur due to the relatively large inter-tube shear strength and small shear stress at the inter-tube surface in this study. Only the calculated SWNT/sheath shear strength from the MD simulation, $18.8 \mathrm{MPa}$, is used for all the case studies. The outer radius of the sheath is assumed to be four times the nanorope radius from finite element 


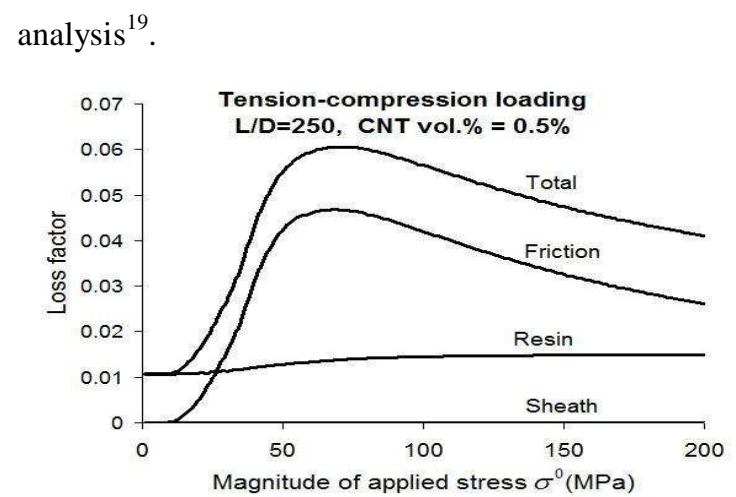

Figure 6. Loss factor for composite with $\theta=0^{\circ}$ aligned nanoropes under tension-compression loading. The loss factor from the viscoelastic sheath cannot be seen on this scale as it is very small-of the order $10^{-5}$ )
The effective loss factor of composites with $\theta=0^{\circ}$ aligned nanoropes under fully-reversed tension-compression cyclic loading, $\sigma=\sigma^{0} \sin (2 \pi t)$, is presented in Fig. 6. The aspect ratio of the nanotube is 250 and the volume fraction of the nanorope is $0.5 \%$. Four lines are plotted with respect to the magnitude of the applied stress in a cycle, $\sigma^{0}$. These lines include the total loss factor, loss factor from the interfacial friction, loss factor from the resin, and loss factor from the sheath as defined in Eq. (9). As shown in Fig. 6, the total effective loss factor and the loss factor from the stick-slip motion are sensitive to the magnitude of the applied stress, while the loss factor of the viscoelastic resin shows only a slight increase with stress. When the applied stress is small (lower than $8 \mathrm{MPa}$ in this case), the nanorope is perfectly bonded to the resin, resulting in a zero loss factor from stickslip motion. As the magnitude of applied stress increases, the debonding at the SWNT/sheath interface occurs and more and more portions of nanorope are debonded from the sheath at the maximum applied stress in a cycle, leading to the increase of total loss factor. Once the applied stress magnitude is larger than certain value, roughly $40 \mathrm{MPa}$ in this case, fully developed debonding occurs in a cycle. This is shown as an inflection point in Fig. 6. The loss factor continues to increase at decreasing rates until an applied stress of $75 \mathrm{MPa}$. Compared to the loss factor from viscoelastic resin and sheath, the loss factor from stick-slip motion dominates when the applied load is large enough to cause the entire interfacial area to slip during the cyclic motion.

The damping behavior of a composite with randomly oriented nanoropes under fully-reversed tensioncompression loading is shown in Fig. 7. The aspect ratio of the nanotube is 250 and the volume fraction of the nanorope is $0.5 \%$. It is seen that $0^{\circ}$ alignment improves the composite's damping ability when interfacial slip is a major contributor to energy dissipation (above $\sim 18 \mathrm{MPa}$ in this example). The loss factor of the composite with aligned nanoropes can be about twice the value of the composite with randomly oriented nanoropes at a certain high stress.

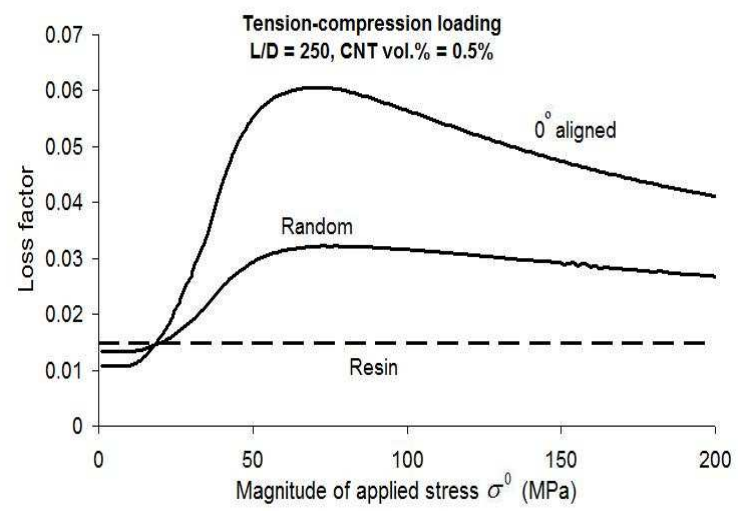

Figure 7. Loss factor of composites with $0^{\circ}$ aligned and in-plane randomly oriented nanoropes under fully-reversed tension- compression loading

\section{Conclusion}

To predict the effective loss factor of composites with dilute SWNT ropes, a multiscale damping model is developed and analyzed. The interfacial shear strengths between the SWNTs and epoxy resin as well as between individual SWNTs are obtained by simulating pullout tests with the molecular dynamics method. These calculated interfacial shear strengths are then applied to a micromechanical damping model to predict SWNT/epoxy composite damping under cyclic loading. The observations of this investigation are summarized as follows:

1) With only van der Waals interactions and with the material system studied, the interfacial shear strength between the nanotubes in a rope is larger than the interfacial shear strength between the nanotubes and the epoxy resin.

2) Due to the interfacial friction between the CNTs and the resin, the loss factor for the composites with nanoropes is sensitive to the applied stress under tension-compression cyclic loading.

3) With a large enough applied stress to cause the entire interfacial area to slip during the cyclic motion, alignment of carbon nanoropes can improve the damping properties for composite with dilute nanoropes. 


\section{Acknowledgments}

The authors acknowledge the financial support of the U.S. Army Research Office (W911NF-07-1-0395).

\section{References}

${ }^{1}$ Zhou, X., Shin, E., Wang, K.W., and Bakis, C.E., "Interfacial Damping Characteristics of Carbon Nanotube Based Composites," Composites Science and Technology, Vol. 64, No. 15, 2004, pp, 2425-2437

${ }^{2}$ Suhr, J., Koratkar, N., Keblinski, P., and Ajayan, P., "Viscoelasticity in Carbon Nanotube Composites," Nature Materials, Vol. 4, No. 2, 2005, pp, 134-137

${ }^{3}$ Buldum, A. and Lu, J.P., “Atomic Scale Sliding and Rolling of Carbon Nanotubes,” Physics Review Letters, Vol. 83, No. 24, 1999, pp, 5050-5053

${ }^{4}$ Hölscher, H., Schwarz, U.D., Zwörner, O., and Wiesendanger, R., "Consequences of the Stick-slip Movement for the Scanning Force Microscopy Imaging of Graphite," Physics Review B, Condensed Matter, Vol. 57, No. 4, 1998, pp, 2477-2481

${ }^{5}$ Zhou, X., Wang, K.W., and Bakis, C.E., "Effects of Interfacial Friction on Composites Containing Nanotube Ropes," Proc. $44^{\text {th }}$ AIAA/ASME/ASCE/AHS /ASC Structures, Structural Dynamics, and Materials Conference, Paper No. AIAA-2004-1784, AIAA, Reston, VA, 2004

${ }^{6}$ Liu, A., Huang, J.H., Wang, K.W., and Bakis, C.E., "Effects of Interfacial Friction on the Damping Characteristics of Composites Containing Randomly Oriented Carbon Nanotube Ropes," Journal of Intelligent Material Systems and Structures, Vol. 17, No. 3, 2006, pp, 217-229

${ }^{7}$ Liu, A., Wang, K.W., Bakis, C.E., Huang, J.H., "Analysis of Damping Characteristics of a Viscoelastic Polymer Filled with Randomly Oriented Single-Wall Nanotube Ropes," Proceeding of SPIE - The International Society for Optical Engineering, Smart Structures and Materials 2006: Damping and Isolation, Vol. 6169, 2006, pp, 616911

${ }^{8}$ Frankland, S.J.V., Bandorawalla, T., Gates, T.S., "Calculation of Non-Bonded Forces due to Sliding of Bundled Carbon Nanotubes," Proc. 44th AIAA/ASME /ASCE/AHS/ASC Structures, Structural Dynamics, and Materials Conference, Vol. 2, AIAA, Reston, VA, 2003, pp, 1252-1262

${ }^{9}$ Tersoff, J., "Modeling Solid-State Chemistry: Interatomic Potentials for Multi-Component Systems," Physical Review B, Vol. 39, No. 8, 1989, pp, 5566-5568

${ }^{10}$ Jorgensen, W.L., Maxwell, D.S., and Tirado-Rives, J., "Development and Testing of the OPLS All-Atom Force Field on Conformational Energetics and Properties of Organic Liquids," Journal of American Chemistry Society, Vol. 118, No. 45, 1996, pp, $11225-11236$

${ }^{11}$ DL_POLY 2.19, the Science Facilities Research Council (STFC), UK, 2008

${ }^{12}$ Barber, A.H., Cohen, S.R., Eitan, A., Schadler, L.S., and Wagner, H.D., "Fracture Transitions at a CarbonNanotube/Polymer Interface," Advanced Materials, Vol. 18, No. 1, 2005, pp, 83-87

${ }^{13}$ Chaturvedi, S.K. and Tzeng, G.Y., "Micromechanical Modeling of Material Damping in Discontinuous Fiber Three-Phase Polymer Composites," Composites Engineering, Vol. 1, No. 1, 1991, pp, 49-60

${ }^{14}$ Shames, I.H. Elastic and Inelastic Stress Analysis, Prentice Hall International Inc., Englewood Cliffs, NJ, 1992

${ }^{15}$ Nelson, D.J. and Hancock, J.W.,"Interfacial Slip and Damping in Fibre Reinforced Composites," Journal of Material Science, Vol. 13, No. 11, 1978, pp, 2429-2440

${ }^{16}$ Lazan, B.J., Damping of Materials and Members in Structural Mechanics, Pergamon Press, Oxford, 1968

${ }^{17}$ Kelly, A., "Interface Effects and the Work of Fracture of Fibrous Composites," Proceeding of the Royal Society of London, Series A, Vol. 319, No. 1536, 1970, pp, 95-116

${ }^{18}$ Odegard, G.M., Gates, T.S., Wise, K.E., Park, C., Siochi, E.J., "Constitutive Modeling of Nanotube-Reinforced Polymer Composites," Composites Science and Technology, Vol. 63, No. 11, 2003, pp, 1671-1687

${ }^{19}$ Liu, A., "Analysis of Damping Characteristics of Polymeric Composites Containing Carbon Nanotubes," Ph.D. Dissertation, Mechanical and Nuclear Engineering Dept, Penn State Univ., University Park, PA, 2009 